\title{
A0/0TA 31-A2 Fractures: Long or Short Gamma Nail?
}

\author{
R Luque*, B Rizo, C Arvinius, Y Lópiz and F Marco \\ Orthopaedic Surgery and Traumatology Department, Hospital Clínico San Carlos, Spain
}

Received: November 10, 2017; Published: November 16, 2017

*Corresponding author: Rafael Luque Pérez, C/Ibiza 15 5ozqda, Spain

\section{Abstract}

Introduction: The Gamma 3 nail is widely used for A031A1-3 fractures. The aim of this study was to compare outcomes in patients with 31A2 fractures treated with two lengths of Gamma3 nail.

Material: A prospective study of 104 non-pathological 31A2 fractures treated January 2012-January 2014. 14 patients were eliminated due to inadequate follow up. Mean follow-up was 18 months (range 12-36). Average age was 81 years (range 50-99), 78\% of the patients were female. We studied two groups: Long Gamma3 Nail (LGN) and Short Gamma3 Nail (SGN). Preoperative variables included: age, medical pathologies, gait, anesthetic risk, associated fractures, hemoglobin and hematocrit values. Postoperatively, we evaluated: functional and radiographic results, quality of life, hemoglobin concentration, hematocrit, transfusion, gait and pain. Intraoperative and postoperative complications were recorded: malunion, nonunion, infection rates, cut-outs and periprosthetic fractures.

Results: We obtained a correct reduction in $73 \%$ of cases. We found two intraoperative complications (greater trochanteric fractures) in two LGN cases. 38\% of the patients with LGN presented nail tip impaction upon the distal anterior femoral cortex, associated with anterior knee pain. Blood loss was statistically different between groups but neither clinical outcomes nor quality of life presented any differences.

Conclusion: Our results with these two sizes of the Gamma3 Nail in 31A2 fractures showed no overall differences in clinical outcomes and complication rates. Despite this, the LGN presented a statistically significant higher decrease of the postoperative hematocrit and more transfused blood concentrates. We therefore recommend the use of locked SGN to treat the 31A2 fractures.

Keywords: Unstable proximal femur fracture; Unstable pertrochanteric femur fracture; Intramedullary nail; Gamma nail

\section{Introduction}

The treatment of proximal femoral fractures is a constant subject of interest due to their high incidence. The present study considers a particular type of fracture characterized by instability, also known as unstable intertrochanteric fracture. This fracture presents a special pattern with a fracture line extending from lateral-proximal to medial-distal affecting the lesser trochanter. The instability of these fractures is exerted by the iliopsoas muscle that inserts in the fractured lesser trochanter and therefore medializes the comminuted postero-medial cortex of the fracture; this reduces the area of contact between the two ends of the fracture, delaying bone callus formation and increasing the risk of implant failure [1]. This fracture's controversy seems to center on the type of treatment used: extramedullary devices versus intramedullary nail [2-5]. The different biomechanical forces involved because the extramedullary devices to produce distraction of the fracture, which leads to a high percentage of implant failures. Few studies have examined the true behavior of intramedullary nails in these fractures, and the existing publications involve a great diversity of nails, thereby complicating interpretation of the results [6-11]. Many publications showed good results of former gamma nails to treat proximal fractures [12-17]. Recently, with the evolution of the gamma nail many studies published and compared the outcomes of the previous generations with the actual Gamma3 [18-24].

In order to study our experience at the Hospital Clinico San Carlos in Madrid, a retrospective study of the treatment of the intertrochanteric fractures with gamma nail was done [12]. In 2000 a total of 348 intertrochanteric femoral fractures, excluding pathological fractures were reviewed. Among the various data collected, the phenomenon of cut out screw was seen in 21 cases, which accounted for $8 \%$ of the series, being therefore the most frequent cause of reoperation. The study of these patients revealed the existence of a combination of factors that could be involved in the failure of the fixation [16]. Some years after, with the new nail generation, we decided to analyze if the results of the long dynamic gamma3 nail (LGN) were comparable to short static gamma nails (SGN) for the treatment of 31A2 fractures in our department.

\section{Materials and Methods}

We present a prospective study of 104 non-pathological unstable trochanteric femoral fractures treated with the third generation 
of Gamma nail @ between January 2012 and January 2014. The patients were aleatorized and divided in 2 groups according to the implant: SGN or LGN (Figure 1). Mean follow-up was 18 months (range 1-3 years). 14 patients were eliminated from the study due to inadequate monitoring (one immediate postoperative death and 13 patients with incomplete record but intact implant in their last visit). The recorded preoperative variables included: patient age, associated diseases, type of gait, anesthetic risk, the presence of associated fractures, hemoglobin and hematocrit concentration and fracture etiology. The preanesthesic risk was collected using the ASA (American Society of Anesthesiologists) classification. The type of gait was classified according to the need of walking aids (cane or walker), the absence of walking ability or the capacity of the patient to walk autonomously without help of any kind. The fractures were classified according to the AO classification (Orthopedic Trauma Association) [6].

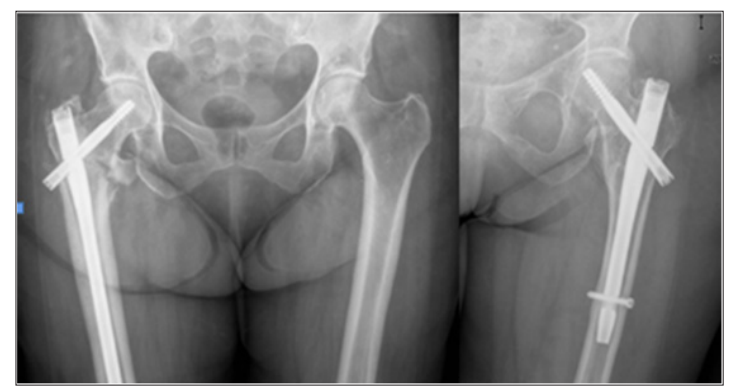

Figure 1: Plain radiographs of unstable pertrochanteric fractures treated with long Gamma 3 nail and short locked Gamma 3 nail.

Postoperatively, we evaluated the following parameters: hemoglobin and hematocrit concentration in the immediate postoperative period, the need for transfusion, the type of gait at discharge from the outpatient clinic and the presence of pain that was scored as follows: no pain, occasional pain, or disabling pain. Functional results and quality of life was queried. Radiographic evaluation was also made including the assessment of fracture reduction and evaluation of cephalic screw location. Intraoperative and postoperative complications were studied; such as malunion, nonunion, infection rates, cut-outs and periprosthetic fractures. Statistical analysis was performed using SPSS 15.0 (SPSS Inc, Chicago Illinois). Student t test was used to compare study outcomes with parametric means. Chi-square test and Fisher's exact test were used to compare non-parametric means. To level of statistical significance was set as a two-sided P value of 0.05 .

\section{Results}

\section{Preoperative Period}

The mean age of the patients was 81 years (range 50-99) and $78 \%$ of the patients were female. The associated medical conditions are summarized in Table 1, and are seen to be very prevalent: $88 \%$ had at least one pathology and $62 \%$ had more than two. By means of the ASA classification: $44 \%$ of the patients corresponded to ASA II, $40 \%$ to ASA III and $16 \%$ were ASA III. The fractures were classified according to the AO classification: 31A2.1: 28\%, 31A2.2: $34 \%$ and the most commonly found 31A2.3: 38\%. We found associated upper limb fractures in $7.1 \%$ of cases (distal radius fractures and proximal humerus fractures). Other associated injuries were seen in $5 \%$ of the cases, the most frequent was traumatic brain injury which was usually mild. The quality of life prior to fracture was: $25 \%$ needed personal care assistance, 33\% were independent at home, 39\% were independent outside their home and only $3 \%$ were able to do physical activities. The type of walking was: $3 \%$ unable to walk, $25 \%$ used two sticks, $38 \%$ used one stick and $34 \%$ walked autonomously. The percentages shown refer to the total of patients, which suggests a very high rate of pathological associations in our patients. The two studied groups were homogeneous and the small differences seen in the distribution were not statistically significant (Table 2).

Table 1: Associated medical conditions.

\begin{tabular}{|c|c|}
\hline & \% \\
\hline None & 12 \\
\hline Digestive & 7 \\
\hline Neurologic & 11 \\
\hline Dementia & 10 \\
\hline Alcohol & 6 \\
\hline Neumologic & 10 \\
\hline Cardiologic & 18 \\
\hline Kidney & 4 \\
\hline AHT & 29 \\
\hline DM & 18 \\
\hline
\end{tabular}

Table 2: Preoperative Study/Implant used.

\begin{tabular}{|c|c|c|c|}
\hline & GN & SGN & LGN \\
\hline ASA I & $0 \%$ & $0 \%$ & $0 \%$ \\
\hline ASA II & $18 \%$ & $20 \%$ & $16 \%$ \\
\hline ASA III & $66 \%$ & $67 \%$ & $64 \%$ \\
\hline ASA IV & $16 \%$ & $13 \%$ & $19 \%$ \\
\hline
\end{tabular}

\section{Surgery}

The patients were operated upon following preanesthetic evaluation, an average of 3 days from admission. Spinal anesthesia was performed in $74 \%$ of cases, epidural anesthesia in $14 \%$ and general anesthesia in $12 \%$. The most commonly used nail was the 130 o cervical-cephalic angled nail (52\%) and the most common length of the cephalic screw was $90 \mathrm{~mm}$ (33\%). There were no significant differences between the distribution of angle and the length of the head screw used between both groups. The diaphyseal locking screw of the long gamma nails was dynamic while the short gamma nails had a static distal locking screw. The average time of surgery was 48 minutes (range 30-68). The only intraoperative complication found was a greater trochanteric fracture in two cases treated with long nails.

\section{Postoperative Hospital Period}

The local complications in the early postoperative period were limited to a single case of a significant hematoma. The most frequent general complications registered during the immediate postoperative period were urinary tract infection (no differences between groups). Mean hemoglobin in the immediate postoperative period was $9 \mathrm{~g} / \mathrm{dl}$ (SGN9.5g/dl,LGN8.5g/dl), whereas the hematocrit 
was $27 \%(29.2 \%$ SGN, $26 \% \mathrm{LGN})$. The blood loss recorded was significantly lower in SGN ( $\mathrm{p}=0.05)$. It was necessary to transfuse an average of 1.4 blood units / patient (SGN0.8, LGN1.65), this difference isn't statistically but clinical significant.

Full limb loading was allowed after 24 hours. The hospital stay was on average 6 days including the preoperative period (5 SGN, 7 LGN). The destinations were elderly care homes, rehabilitation centers or secondary hospitals in $65 \%$ of cases and the patient's home in $35 \%$ of cases. No significant differences were found in hospital stay or destinations.

\section{Outpatient clinic}

The mean time to fracture consolidation was 3.1 months without differences between groups. To be considered as consolidation an evident bone callus formation had to be present of both the anteroposterior and axial projection. Fracture reduction was evaluated in the postoperative x-ray. In 73\% a correct reduction of the fracture was obtained (73\% SGN, 74\% LGN), 18.5\% of the cases didn't have medial cortex contact ( $21 \%$ SGN, $13 \%$ LGN), in $7 \%$ the lateral reduction was unsatisfactory (SGN 6\%, LGN 10\%) while $1,5 \%$ presented a complete lack of reduction (SGN 0\%, LGN 3\%), no significant differences were found. The placement of the cephalic screw was studied in both the anteroposterior radiographs and in the axial, without significant differences between the locations in both groups. In all cases treated with SGN the cephalic screw was placed in the two inferior thirds of the head and neck as in $97 \%$ of the LGN. Concerning the axial x-ray, both SGN and LGN head screws were placed in the posterior part of the head. The mean screwsubchondral bone distance was $9 \mathrm{~mm}$ (range $4-20 \mathrm{~mm}$ ). 38\% of the patients with LGN presented nail tip impaction upon the distal anterior cortical of the femur, this radiographic impaction was associated with pain in the anterior zone of the knee (Figure 2).

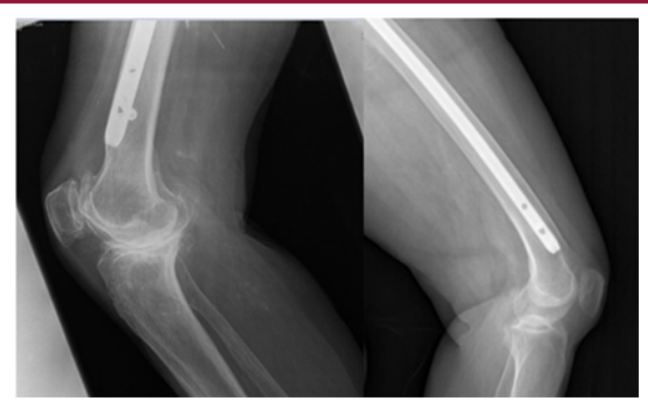

Figure 2: Plain radiographs showing a long Gamma Nail tip impaction upon the distal anterior cortical of the femur.

Since this situation is not to be found in the SGN group, it was a significant difference between the groups. There were not any statistically significant differences between groups concerning the late complications studied, even though they were more frequent in the LGN group. We found one case of delayed consolidation that required surgery revision with replacement of the LGN. We also had one LGN with cephalic screw lateral protrusion two months after surgery. Loading of the affected limb was avoided until complete consolidation was obtained and the material was finally extracted. Finally we found one SGN case of cephalic screw cut-off that required extraction and hip arthoplasty (Figure 3). The patients were discharged from the outpatient clinic after an average follow- up of 24.3 weeks (SGN 24, LGN 25, no differences found). Before the discharge the functional results were evaluated. The type of gait at discharge from the clinic is reported in Table 3. Sixty-eight percent of the patients were free of pain, however, $15 \%$ of the LGN reported discomfort in the anterior zone of the knee, $2 \%$ of these patients referred a disabling pain. In all cases this coincided with impaction of the nail on the distal anterior cortical layer of the femur but only 3 cases underwent surgery with removal of the implant. There were no statistically significant differences between groups about the lower limb pain. It was described as disabling by only $2 \%$ of all the patients.

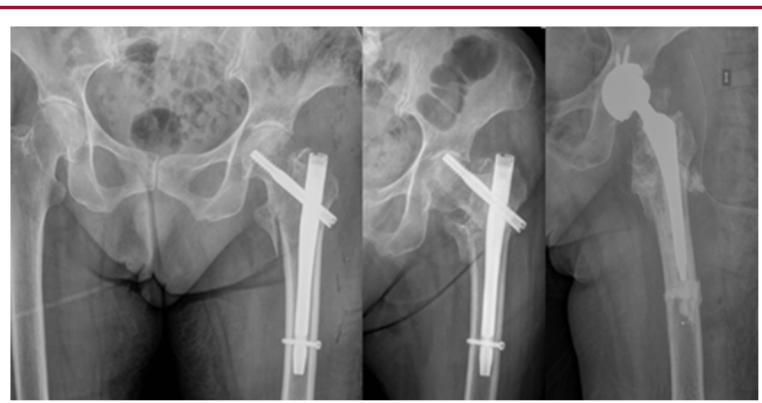

Figure 3: Plain radiographs showing a case with cephalic screw cut-off that ended in arthroplasty.

Table 3: Gait pre- and post-operatively.

\begin{tabular}{|c|c|c|}
\hline & Pre & Post \\
\hline Don't walk & $4 \%$ & $11 \%$ \\
\hline Walker & $1 \%$ & $3 \%$ \\
\hline Two canes & $2 \%$ & $5 \%$ \\
\hline One cane & $34 \%$ & $42 \%$ \\
\hline Independent & $59 \%$ & $21 \%$ \\
\hline
\end{tabular}

\section{Discussion}

The results obtained with the two sizes of the Gamma3 Nail in unstable pertro chanteric femoral fractures did not show any important differences in neither clinical outcomes nor complication rates. Theses unstable fractures, also called 31A2 fractures account for $37-42 \%$ of all intertrochanteric fractures of the femoral region $[12,16]$. The treatment of these fractures is subject to controversy, particularly concerning the use of intramedullary versus extramedullary devices. However, there is one point on which the different studies seem to agree: extramedullary sliding devices (e.g., SHS) are not adequate for fractures of this kind [25]. As a result of their distinct biomechanics, these devices distract rather than compress the fracture fragments thus leading to a high percentage of failures. The great diversity of implants used within the same study contributes to perplex the situation. In series such as those published by Parker, Chinzei, Barton, Michael or Cheng, as many as four different types of nails have been used [6-11]. In our study we have only used the Gamma3 nail (Stryker Howmedica), a fact that allows us to adequately define its behavior. In unstable fractures, intramedullary nailing theoretically affords increased stability since the leverage is less and the proximal portion of the nail is supported against the proximal fragment. In our series, the percentage of implant failure $(0,7 \%$ cutting phenomenon, $0,7 \%$ nail rupture and $0,7 \%$ pseudoarthrosis) was considerably lower 
than with extramedullary fixation and slightly lower than other published intramedullary series [8,9].

Many studies have used intramedullary nails for the treatment of unstable trochanteric fractures [6-11]. On one hand the problem of these studies is that unstable fractures are not only A.2 fractures but also A1.3 and inverse fracture patterns (A3.1, A3.3) and on the other hand the series where only the A.2 fracture was contemplated, the patient series were of limited in size [14]. The advantages of the endomedullary nail are lesser blood loss and a lower percentage of failures, the latter being essential in order to avoid reinterventions that pose an important risks for the elderly patient $[12,15,17]$. On the other hand, the consolidation rate obtained is high (with only one case of pseudoarthrosis in our series), and with the use of short nails one of the most common complications in endomedullary nailing procedures is diminished: diaphyseal refracture fundamentally associated with the use of long gamma-nails (not seen in our series) [13]. Failure of closed fracture reduction is relatively infrequent, since perfect anatomical reduction is not necessary for implantation, the consolidation can be achieved provided that there is sufficient contact between the bone fragments. However, we agree that a positive medial cortical support allows limited sliding of the neck fragment and achieve secondary stability [8].

This leads to clinical-radiological discordance in many cases. The need to access the fracture focus is only considered in those cases where all the non-aggressive reduction options have been exhausted, and the open reduction might be related to pseudoarthrosis and intraoperative fractures. We agree with Pervez [14] and López-Vega [19] concerning the distal nail locking to get additional stabilization to the nail. In $13 \%$ of the patients the nail impacted upon the distal anterior cortical layer of the femur. This situation is more than a simple radiological finding, since in contradiction to others authors, impaction caused clinical manifestations in our patients (pain in the anterior knee region). This circumstance could be related to the characteristics of the femur in our elderly population, which are different to those found in the central European population [15]. The decision to adopt the Gamma3 Nail as our implant in this type of fractures was based on the findings of last year's studies between the previous gamma-nail and the Gamma3, in which the Gamma3 afforded the best results [18-24]. We agree with most authors that the best way to prevent complications is to ensure a careful surgical technique, with special attention to medullary cavity drilling and the manual insertion of the nail [13]

In our study we recorded a single cutting phenomenon, in contrast to the $6 \%$ rate reported in another series reviewed in our hospital, involving 348 trochanteric fractures treated with the short gamma-nail, and where $37 \%$ were A.2 type fractures. This could, in part, be related to an improved surgical technique as well as the increased stability afforded by the long gamma3-nail, due to its lesser leverage and increased length [16]. There are no publications to be found that studies the difference between two different types of the same implant. Although our study are not enough to rise statistically significant differences between the groups some are clinical significant. The data obtained allow us to affirm that the clinical and radiological results with SGN and the LGN in the treatment of the AO/OTA 31A2 proximal femur fractures are similar. We found that there are statistically significant differences of transfused blood concentrates and higher decrease in the postoperative hematocrit, the LGN caused more blood loss and could there for potentially cause major medical complications. We found differences in favor of the use of SGN opposite the LGN, such as the presence of pain in the distal third of the femur as some late complications.

\section{References}

1. Parker MJ (1996) Trochanteric hip fracture, Fixation failure componer with femoral edialisation, a comparison of 101 cases. Acta Orthop Scand 67: 329-332.

2. Parker MJ, Handoll HH (2000) Gamma and other cephalocondylic intramedullary nails versus extramedullary implants for extracapsular hip fractures, Cochrane Database Syst Rev 2: 293-299.

3. Chinzei N, Hiranaka T, Niikura T, Tsuji M, Kuroda R, et al. (2015) Comparison of the Sliding and Femoral Head Rotation among Three Different Femoral Head Fixation Devices for Trochanteric Fractures. Clin Orthop Surg 7(3): 291-297.

4. Barton TM, Gleeson R, Topliss C, Greenwood R, Harries WJ, et al. (2010) A comparison of the long gamma nail with the sliding hip screw for the treatment of A0/OTA 31-A2 fractures of the proximal part of the femur: a prospective randomized trial. J Bone Joint Surg Am 92(4): 792-798.

5. Baumgaertner MR, Curtin SL, Lindskog DM (1998) Intramedullary versus extramedullary fixation for the treatment of intertrochanteric hip fractures. Clin Orthop 348: 87-94.

6. Michael D, Yaniv W, Tal FR, Kessler Evan G, Eyal A, et al. (2015) Expandable proximal femoral nail versus gamma proximal femoral nail for the treatment of AO/OTA 31A1-3 fractures. Injury 15: 615-629.

7. Andreani L, Bonicoli E, Piolanti N, Niccolai F, Carmignani A, et al. (2015) Prospective Randomized Controlled Trial of Two Different Intramedullary Nails for Pertrochanteric Fractures of the Femur. Surg Technol Int 27: 210-214.

8. Chang SM, Zhang YQ, Ma Z, Li Q Dargel J, et al. (2015) Fracture reduction with positive medial cortical support: a key element in stability reconstruction for the unstable pertrochanteric hip fractures. Arch Orthop Trauma Surg 135(6): 811-818.

9. Xu Y, Geng D, Yang H, Wang X, Zhu G (2010) Treatment of unstable proximal femoral fractures: comparison of the proximal femoral nail antirotation and gamma nail 3. Orthopedics 33(7): 473.

10. Winnock de Grave P, Tampere T, Byn P, Van Overschelde J, Pattyn C, et al. (2012) Intramedullary fixation of intertrochanteric hip fractures: a comparison of two implant designs. A prospective randomized clinical trial. Acta Orthop Belg 78(2): 192-198.

11. Ceder L (2000) The difficult extracapsular hip fracture (including subtrochanteric). Current Orthopaedics 14(2): 93-101.

12. Vicario C, Marco F, Domínguez I, López-Durán L (2000) Tratamiento en el anciano de las fracturas de la región trocantérea femoral con clavo intramedular gamma. Rev Ortop y Traumatol 5: 434-438.

13. Barquet A, Francescoli L, Rienzi D, López L (2000) Intertrochantericsubtrochanteric fractures: treatment with the long gamma nail. J Orthop Trauma 14(5): 324-328.

14. Pervez H, Parker MJ (2001) Results of the long Gamma nail for complex proximal femoral fractures. Injury 32(9): 704-707.

15. Anchuela J, Carretero R, San Martín A et al. (2002) El clavo Gamma largo en las fracturas extracapsulares del fémur. Rev Ortop y Traumatol 6: 234-238. 
16. Vicario Espinosa C, Marco Martínez F, Ortega L, Alcobendas Maestro M, Dominguez I, et al. (2003) Necrosis of the femoral head after fixation of throchanteric fractures with Gamma locking nail. A cause of late mechanical failure Injury 34: 129-134.

17. Li Z, Liang Y, Zhao C, Wu H (2014) Treatment of elderly femoral intertrochanteric fractures with long Gamma nail 94(43): 3436-3438.

18. Heiney J, Battula S, Njus G, Ruble C, Vrabec G (2008) Biomechanical comparison of three second-generation reconstruction nails in an unstable trochanteric femur fracture model. Proc Inst Mech Eng $\mathrm{H}$ 222(6): 959-966.

19. López-Vega M, Gil-Monzó ER, Rodrigo-Pérez JL, López-Valenciano J, Salanova-Paris RH, et al. (2015) Randomized prospective study on the influence distal block and Gamma 3 nail on the treatment of intertrochanteric fractures of femur. Rev Esp Cir Ortop Traumatol 59(1): 26-35.

20. Mingo-Robinet J, Torres-Torres M, Martínez-Cervell C, Alonso Del Olmo JA, Rivas Laso JA, et al. (2015) Comparative study of the second and third generation of gamma nail for trochanteric fractures: review of 218 cases. J Orthop Trauma 29(3): 85-90.

21. Huang H, Xin J, Ma B (2014) Analysis of complications of intertrochanteric fracture treated with Gamma 3 intramedullary nail. Int J Clin Exp Med $7(10): 3687-3693$.

22. Georgiannos D, Lampridis V, Bisbinas I (2014) Complications following Treatment of Trochanteric Fractures with the Gamma3 Nail: Is the Latest Version of Gamma Nail Superior to Its Predecessor? Surg Res Pract 14: 1435-1439.

23. Kempf I, Grosse A, Taglang G, Favreul E (2014) Gamma nail in the treatment of closed trochanteric fractures. Results and indications of 121 cases. Orthop Traumatol Surg Res 100(1): 75-83.

24. Westacott D, Bould M (2011) Outcome in 36 elderly patients treated with the Gamma3 Long Nail for unstable proximal femoral fracture. Acta Orthop Belg 77(1): 68-772.

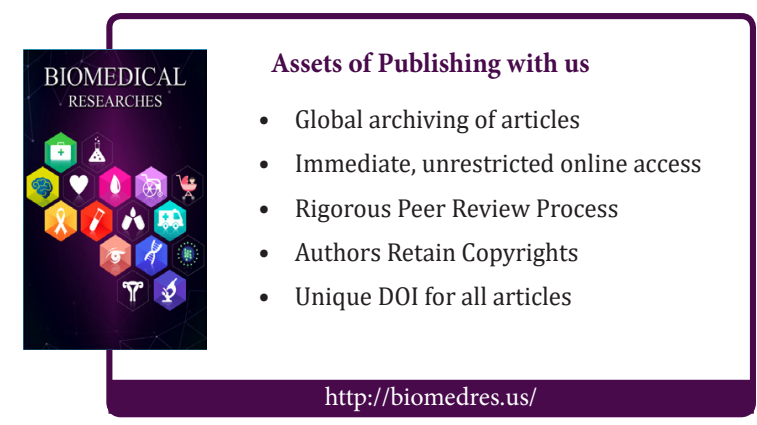

